\title{
Non-Interactive Key Exchange
}

\author{
Eduarda S.V. Freire ${ }^{1, \star}$, Dennis Hofheinz, ${ }^{2, \star \star}$, Eike Kiltz ${ }^{3, \star \star \star}$, \\ and Kenneth G. Paterson ${ }^{1, \dagger}$ \\ 1 Royal Holloway, University of London \\ ${ }^{2}$ Karlsruhe Institute of Technology \\ 3 Ruhr-Universität Bochum
}

\begin{abstract}
Non-interactive key exchange (NIKE) is a fundamental but much-overlooked cryptographic primitive. It appears as a major contribution in the ground-breaking paper of Diffie and Hellman, but NIKE has remained largely unstudied since then. In this paper, we provide different security models for this primitive and explore the relationships between them. We then give constructions for secure NIKE in the Random Oracle Model based on the hardness of factoring and in the standard model based on the hardness of a variant of the decisional Bilinear Diffie Hellman Problem for asymmetric pairings. We also study the relationship between NIKE and public key encryption (PKE), showing that a secure NIKE scheme can be generically converted into an IND-CCA secure PKE scheme. Our conversion also illustrates the fundamental nature of NIKE in public key cryptography.
\end{abstract}

Keywords: non-interactive key exchange, public-key cryptography, pairings.

\section{Introduction}

Non-interactive key exchange (NIKE) is a cryptographic primitive which enables two parties, who know each others' public keys, to agree on a symmetric shared key without requiring any interaction. The canonical example of a NIKE scheme can be found in the seminal paper by Diffie and Hellman [1]: let $G$ be a group of prime order $p$ with generator $g$, and assume Alice has public key $g^{x} \in G$ and private key $x \in \mathbb{Z}_{p}$, while Bob has public key $g^{y} \in G$ and private key $y \in \mathbb{Z}_{p}$. Then Alice and Bob can both compute the value $g^{x y} \in G$ without exchanging any messages. More properly, Alice and Bob should hash this key together with their identities in order to derive a symmetric key $H\left(\mathrm{Alice}, \mathrm{Bob}, g^{x y}\right)$.

* Eduarda S.V. Freire was supported by CAPES Foundation/Brazil on grant 0560/09-0 and Royal Holloway, University of London.

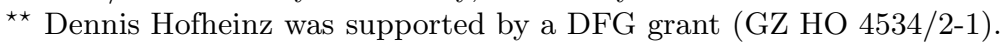

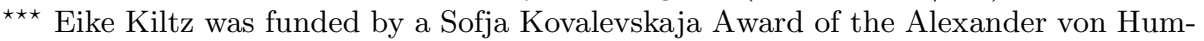
boldt Foundation and the German Federal Ministry for Education and Research.

${ }^{\dagger}$ Kenneth G. Paterson was supported by EPSRC Leadership Fellowship $\mathrm{EP} / \mathrm{H} 005455 / 1$. 
This example encapsulates in a nutshell all the basic features required of a NIKE scheme: users should agree on some common parameters ( $p, G$ and $g$ here), then create their key pairs. Once these are computed and the public keys distributed, any pair of users can set up a shared key without further exchange of messages. The security properties desired of NIKE are, informally at least, clear: compromise of one user's private key should not affect the security of shared keys between pairs of uncorrupted users; compromise of one shared key should not undermine the security of other shared keys. Naturally, since the primitive is noninteractive, one cannot hope to obtain any kind of forward security properties. In practice, the public keys will be certified, and consideration needs to be given to modelling the key registration process.

NIKE has real-world applications. In wireless and sensor networks, conserving battery power is a prime concern, and so the energy cost of communication must be minimised. Thus using key establishment methods that minimise the number of bits that need to be transmitted is of fundamental importance. In particular, when faced with a jamming adversary, reducing the total number of rounds of interaction needed to establish a key is particularly helpful. NIKE is an excellent option in solving this problem, since a key can be established with minimal communication and interaction: assuming the public keys are pre-distributed, all that is needed is an exchange of identifiers for those keys, and often this exchange must take place anyway, in order to establish communications. A recent paper 2] gives a detailed evaluation of the energy costs of interactive and non-interactive key exchange protocols in the ID-based and PKI settings for wireless communications with a jamming adversary, demonstrating that significant energy savings can be made by adopting a non-interactive approach to key establishment. Its non-interactive nature makes NIKE an abstract building block that is qualitatively different from interactive key exchange: e.g., to achieve deniable authentication, 3] explicitly requires a non-interactive key exchange. But NIKE can also be used as a basis for interactive key exhange [4]: one can use the shared key in a MAC to authenticate an exchange of ephemeral DiffieHellman values. Finally, NIKE can be used to build very simple non-interactive designated verifier signature schemes [5], again using the shared key in a MAC to authenticate messages. Thus NIKE appears in various guises throughout the literature.

Despite its appearing in the very first paper on public key cryptography, the NIKE primitive has so far received scant attention as a primitive in its own right. Cash, Kiltz and Shoup (CKS) [6] provided a basic security model for NIKE and analysed the Diffie-Hellman-based scheme above, as well as a twinned variant of it, in the Random Oracle Model (ROM). There is also some work in the ID-based setting [7/910], also all restricted to the ROM.

Our Contributions: Our contention is that NIKE is long overdue for more serious attention and development. In this paper, we initiate the systematic study of NIKE in the public key setting, providing: models and their relationships; constructions for secure NIKE in the Random Oracle Model and in the standard model in the challenging setting where the adversary can introduce arbitrary 
public keys into the system; and a construction for IND-CCA secure public key encryption (PKE) from any secure NIKE. Let us expand on each of these contributions in turn.

Models: It would seem that definitions and security models for interactive key exchange (e.g., 11121314) could provide a natural starting point for formalising NIKE. However, here we take the CKS definition [6] for NIKE as our starting point. One reason for using a case-tailored NIKE definition is simplicity: existing security models for interactive key exchange give considerable attention to properties which are irrelevant in the NIKE setting. (For instance, forward security, multiple sessions, and in particular the pairing of sessions play no role in a non-interactive setting.) Another reason for a case-tailored NIKE definition is that we can focus on adversarial key registration queries; these are usually only implicitly [14] (or not at all [1113]) considered in the standard models for interactive key exchange1. However, in our setting, adversarial key registrations pose the main technical obstacle to achieve NIKE security, as we will explain below.

The CKS security model for NIKE uses an indistinguishability- and gamebased approach to define security, with the adversary being required to distinguish real from random keys in responses to its test queries. The model does allow the adversary to register public keys of his choice in the system and then to make queries for the shared keys between these "corrupted" users and honest (non-adversarially controlled) users, so-called corrupt reveal queries. This translates in the real world to minimising the assumptions made about certification procedures followed by the Certification Authority (CA) in the PKI supporting the NIKE: it means that the CA is not assumed to check that a public key submitted for certification has not been submitted before, and does not check that the party submitting the public key knows the corresponding private key. The model for NIKE in [6] is similar to, and presumably inspired by, the early work of Shoup [12] on interactive key exchange, where capturing so-called PKI attacks, also known as rogue-key attacks, was intrinsic to the security modelling. This modelling approach is referred to elsewhere in the literature as the plain setting (see [1617] and the references therein) or the bare PKI setting [3]. The CKS model is certainly more challenging than settings where proofs of knowledge or proofs of possession of private keys are assumed to be given during registration, or where the adversary must reveal its secret key directly (as with the knowledge of secret key assumption used in [18 19]). However, the CKS model has some shortcomings: the adversary is not allowed to directly query for the shared keys held between pairs of honest users, but instead only gets to see real or random values for these via test queries. Moreover the model does not allow an adversary to query for the private keys of honestly registered users.

Therefore, as a necessary precursor to the further development of NIKE, we start by exploring different models for NIKE and their relationships (Section 2).

\footnotetext{
${ }^{1}$ We mention that some security analyses (e.g., [15]) and Shoup's security model [12]
} do explicitly consider adversarial key registration queries. 
In summary, we introduce three new security models for NIKE and show that they are all polynomially equivalent to one another and to the original CKS model from [6]. One of our models, the $m$-CKS-heavy model, augments the CKS model and effectively allows all conceivable queries, without allowing the adversary to win trivially. It is our preferred security model for NIKE. Another of our models, CKS-light, allows only two honest users, no corruption of honest users, and a single test query. Thus it is particularly simple and so easy to use when analyzing specific NIKE schemes; moreover our results showing equivalence between the models ensure that security in this model implies security in the preferred $m$-CKS-heavy model.

We stress that all these models allow the adversary to register public keys of his choice in the system, so are in the plain setting.

Constructions for NIKE: In Section 4, we give two concrete constructions for NIKE schemes meeting our CKS-light security definition, and hence secure in our preferred $m$-CKS-heavy model (with dishonest key registrations).

Our two constructions are inspired by public key encryption (PKE) schemes which are secure against chosen-ciphertext attacks (IND-CCA secure). We note that dealing with corrupt reveal queries requires techniques to guard against active attacks, which in part explains the connection to IND-CCA security. Indeed, we will also show how to go in the reverse direction, converting any secure NIKE scheme into an IND-CCA secure PKE scheme, see below. We stress, however, that we cannot simply take any IND-CCA secure PKE scheme and directly interpret it as a NIKE scheme 2 Rather, our constructions for NIKE exploit specific properties of the underlying PKE schemes. In fact, our belief is that a generic construction for secure NIKE from PKE is unlikely to be forthcoming.

The first scheme acts as a warm-up. It is provably secure under the factoring assumption in the Random Oracle Model (ROM) and uses ideas from 20] to analyse the basic Diffie-Hellman scheme, where keys are of the form $H\left(\right.$ Alice, Bob, $\left.g^{x y}\right)$, in the group of signed quadratic residues. We note that closely related schemes were analysed in [6], but in different groups and under different assumptions. Specifically, a twinned version of the scheme was proved secure under the CDH assumption, while it is stated that the basic Diffie-Hellman scheme is secure under the Strong DH assumption.

We remark that the latter claim of [6] is problematic. Concretely, the Strong DH assumption is not (directly) sufficient to show that the basic Diffie-Hellman scheme is secure. Namely, the corresponding security reduction requires two $\mathrm{DDH}$ oracles - one for each of the two users sharing the key on which the adversary wants to be challenged - while the Strong DH assumption supplies only one. Certainly this problem could be solved instead by appealing to a suitable gap-DH assumption. We show how to overcome this problem in the group

\footnotetext{
${ }^{2}$ One reason is that it is not clear what should correspond to the NIKE public key: a PKE public key, a PKE ciphertext, or a combination of both? Besides, the corresponding security experiments for NIKE and PKE schemes are rather different: there usually is one challenge ciphertext in a PKE security experiment, while there are at least two challenge users in a NIKE security experiment.
} 
of signed quadratic residues without the need to rely on a gap assumption. We then proceed to sketch how to transport this scheme to the standard model, under the additional assumption that the adversary only registers valid public keys. Because of the extra assumption, this scheme does not strictly speaking meet our security definitions, and would require validity to be enforced by some means in an interactive registration protocol (for example, via a proof of correctness of the public key). This limitation of our standard model, factoring-based solution reflects the technical challenge involved in achieving our "bare PKI" security notions.

Our second NIKE scheme is provably secure in the standard model and combines a specific weak Programmable Hash Function [21 whose output lies in a pairing group and a Chameleon hash function [22]. This enables the simulation in our security proof for the scheme to handle the tricky queries for shared keys involving an honestly generated public key and an adversarially chosen public key. Similar ideas were used in the context of HIBE in [23]. We also make use of the pairing to provide a means of checking that public keys coming from the adversary are in some sense well-formed. We work with asymmetric pairings for efficiency at high security levels (and because it does not add any real complexity to the description of our scheme). The scheme's security relies on a natural variant of the Decisional Bilinear Diffie-Hellman (DBDH) assumption for the asymmetric setting.

From NIKE to PKE: In Section 5, we explore the connections between NIKE and public key encryption (PKE). That such connections exist should not be too much of a surprise: it is folklore that the ElGamal encryption scheme 24] can be seen as arising from the Diffie-Hellman NIKE scheme by making the sender's key pair $\left(g^{x}, x\right)$ ephemeral and using the receiver's public key $g^{y}$ to create the basis for a shared key $g^{x y}$. Similar connections were explored in the ID-based setting in [10].

In our setting with dishonest key registrations, we provide a simple, generic construction for PKE from NIKE that is also in the spirit of the original DiffieHellman-to-ElGamal conversion. The construction takes a NIKE scheme that is secure in our CKS-light model (with dishonest key registrations) and a strongly one-time secure signature scheme as inputs, and produces from these components a Key Encapsulation Mechanism (KEM) that we prove to be IND-CCA secure. A secure PKE from such a KEM can be obtained using standard results. At a high level, the key pair for the KEM is a randomly generated key pair $(p k, s k)$ from the NIKE scheme, ciphertexts are also randomly generated public keys $p k^{\prime}$ from the NIKE scheme (together with a one-time signature that binds the public key to an identity), while the encapsulated key is the shared key computed from $s k^{\prime}$ and $p k$; the receiver computes the same key from $s k$ and $p k^{\prime}$, assuming the one-time signature verifies. In order to prove the KEM to be IND-CCA secure, we exploit the presence of corrupt reveal queries in the NIKE security model in an essential way to handle certain decapsulation queries. The resulting KEM is almost as efficient as the underlying NIKE scheme. 
The fact that secure NIKE implies IND-CCA-secure PKE, one of the most important primitives in cryptography, illustrates the fundamental role and utility of NIKE. We believe that this connection should spur further research on the topic.

\section{Non-Interactive Key Exchange and Security Models}

\subsection{Non-Interactive Key Exchange}

Following [6], we formally define a Non-Interactive Key Exchange (NIKE) scheme in the public key setting to be a collection of three algorithms: CommonSetup, NIKE. KeyGen and SharedKey together with an identity space $\mathcal{I D S}$ and a shared key space $\mathcal{S H K}$. Note that identities in the scheme and security model are merely used to track which public keys are associated with which users - we are not in the identity-based setting.

- CommonSetup: On input $1^{k}$, outputs params, a set of system parameters.

- NIKE. KeyGen: On input params and an identity ID $\in \mathcal{I D} \mathcal{S}$, outputs a public key/secret key pair $(p k, s k)$. This algorithm is probabilistic and can be executed by any user. We assume, without loss of generality, that params is included in $p k$.

- SharedKey: On input an identity $\mathrm{ID}_{1} \in \mathcal{I D} \mathcal{S}$ and a public key $p k_{1}$ along with another identity $\mathrm{ID}_{2} \in \mathcal{I D S}$ and a secret key $s k_{2}$, outputs either a shared key in $\mathcal{S H K}$ for the two identities, or a failure symbol $\perp$. This algorithm is assumed to always output $\perp$ if $\mathrm{ID}_{1}=\mathrm{ID}_{2}$.

For correctness, we require that, for any pair of identities $\mathrm{ID}_{1}, \mathrm{ID}_{2}$, and corresponding key pairs $\left(p k_{1}, s k_{1}\right)$ and $\left(p k_{2}, s k_{2}\right)$, algorithm SharedKey satisfies the constraint:

$$
\operatorname{SharedKey}\left(\mathrm{ID}_{1}, p k_{1}, \mathrm{ID}_{2}, s k_{2}\right)=\operatorname{SharedKey}\left(\mathrm{ID}_{2}, p k_{2}, \mathrm{ID}_{1}, s k_{1}\right)
$$

\subsection{Definitions of Security for Non-Interactive Key Exchange}

Cash, Kiltz and Shoup [6] proposed a security model for NIKE schemes in the public key setting, denoted here by the $C K S$ model. This model abstracts away all considerations concerning certification and PKI in a particularly nice way. It allows an adversary to obtain honestly generated public keys, but also to then associate such public keys with other identities, and to register dishonestly generated public keys (for which the adversary need not know the corresponding private keys). This dishonest key registration (DKR) setting (abstractly) models a PKI where minimal assumptions are made about the actions of the Certificate Authority (CA): the CA is not assumed to check that a public key has not been previously registered to another user, and does not demand a proof of knowledge or possession of the private key when issuing a certificate on a public key. This conservative approach to modelling is fully appropriate given the great diversity 
in how CAs operate in the real world. The model can be seen as a natural adaptation of the approach of Shoup [12] for modelling interactive key exchange to the NIKE setting and is analogous to the plain setting studied in [1617.

However, there are some obvious omissions from the model, including the ability of an adversary to "corrupt" honestly generated public keys to learn the corresponding private keys, and the ability of a user to directly learn the key shared between two honest parties in the system (which could be possible, for example, because of cryptanalysis of a scheme making use of the shared key). Equivalent queries in the ID-based setting were permitted in the model introduced in [10].

For this reason, we augment the original $C K S$ model with the "missing" queries, introducing the $m$-CKS-heavy model. We regard this as providing the "correct" model for NIKE. We also introduce two further models, the CKSheavy and $C K S$-light models. These differ from $m$-CKS-heavy and the original $C K S$ model only in the numbers and types of query that the adversary is allowed to make. Next we present in detail the $m$-CKS-heavy model. Then in Table 1 we summarize the differences between these security models in the DKR setting.

The $m$-CKS-heavy model: Our model is stated in terms of a game between an adversary $\mathcal{A}$ and a challenger $\mathcal{C}$. In this game, $\mathcal{C}$ takes as input the security parameter $1^{k}$, runs algorithm CommonSetup of the NIKE scheme and gives $\mathcal{A}$ params. The challenger takes a random bit $b$ and answers oracle queries for $\mathcal{A}$ until $\mathcal{A}$ outputs a bit $\hat{b}$. The challenger answers the following types of queries for $\mathcal{A}$ :

- Register honest user ID: $\mathcal{A}$ supplies an identity ID $\in \mathcal{I D S}$. On input params and ID, the challenger runs NIKE.KeyGen to generate a public key/secret key pair $(p k, s k)$ and records the tuple (honest, ID, $p k, s k$ ). The challenger returns $p k$ to $\mathcal{A}$.

- Register corrupt user ID: In this type of query, $\mathcal{A}$ supplies both an identity ID $\in \mathcal{I D S}$ and a public key $p k$. The challenger records the tuple (corrupt, ID $, p k, \perp$ ). We stress that $\mathcal{A}$ may make multiple "Register corrupt user ID" queries for the same ID during the experiment. In that case, only the most recent (corrupt, ID, $p k, \perp$ ) entry is kept.

- Extract queries: Here $\mathcal{A}$ supplies an identity ID that was registered as an honest user. The challenger looks for a tuple (honest, ID, $p k, s k$ ) containing ID and returns $s k$ to $\mathcal{A}$.

- Reveal queries: Here $\mathcal{A}$ supplies a pair of registered identities $\mathrm{ID}_{1}, \mathrm{ID}_{2}$, subject only to the restriction that at least one of the two identities was registered as honest. The challenger runs SharedKey using the secret key of one of the honest identities and the public key of the other identity and returns the result to $\mathcal{A}$. Note that here the adversary is allowed to make reveal queries between two users that were originally registered as honest users. We denote by honest reveal the queries involving two honest users and by corrupt reveal the queries involving an honest user and a corrupt user. 
Table 1. Types of queries for different security models in the dishonest key registration (DKR) PKI model (aka plain/bare model). Notation: $\checkmark$ means that an adversary is allowed to make an arbitrary number of queries; $\boldsymbol{X}$ means that no queries can be made; numbers represent the number of queries allowed to an adversary.

\begin{tabular}{lcccccc}
\hline Model & $\begin{array}{c}\text { Register } \\
\text { Honest }\end{array}$ & $\begin{array}{c}\text { Register } \\
\text { Corrupt }\end{array}$ & Extract & $\begin{array}{c}\text { Honest } \\
\text { Reveal }\end{array}$ & $\begin{array}{c}\text { Corrupt } \\
\text { Reveal }\end{array}$ & Test \\
\hline$C K S$-light & 2 & $\checkmark$ & $\boldsymbol{X}$ & $\boldsymbol{X}$ & $\checkmark$ & 1 \\
$C K S$ & $\checkmark$ & $\checkmark$ & $\boldsymbol{x}$ & $\boldsymbol{x}$ & $\checkmark$ & $\checkmark$ \\
$C K S$-heavy & $\checkmark$ & $\checkmark$ & $\checkmark$ & $\checkmark$ & $\checkmark$ & 1 \\
$m$-CKS-heavy & $\checkmark$ & $\checkmark$ & $\checkmark$ & $\checkmark$ & $\checkmark$ & $\checkmark$ \\
\hline
\end{tabular}

- Test queries: Here $\mathcal{A}$ supplies two distinct identities $\mathrm{ID}_{1}, \mathrm{ID}_{2}$ that were both registered as honest. The challenger returns $\perp$ if $\mathrm{ID}_{1}=\mathrm{ID}_{2}$. Otherwise, it uses the bit $b$ to answer the queries. If $b=0$, the challenger runs SharedKey using the public key for $\mathrm{ID}_{1}$ and the secret key for $\mathrm{ID}_{2}$ and returns the result to $\mathcal{A}$. If $b=1$, the challenger generates a random key, records it for later, and returns that key to the adversary. In this case, to keep things consistent, the challenger returns the same random key for the pair $I_{1}, I_{2}$ every time $\mathcal{A}$ queries for their paired key, in either order.

$\mathcal{A}$ 's queries may be made adaptively and are arbitrary in number. To prevent trivial wins for the adversary, no query to the reveal oracle is allowed on any pair of identities selected for test queries (in either order), and no extract query is allowed on any of the identities involved in test queries. Also, we demand that no identity registered as corrupt can later be the subject of a register honest user ID query, and vice versa.

When the adversary finally outputs $\hat{b}$, it wins the game if $\hat{b}=b$. For an adversary $\mathcal{A}$, we define its advantage in this security game as:

$$
\operatorname{Adv}_{\mathcal{A}}^{\text {m-CKS-heavy }}\left(k, q_{H}, q_{C}, q_{E}, q_{H R}, q_{C R}, q_{T}\right)=|\operatorname{Pr}[\hat{b}=b]-1 / 2|
$$

where $q_{H}, q_{C}, q_{E}, q_{H R}, q_{C R}$ and $q_{T}$ are the numbers of register honest user ID queries, register corrupt user ID queries, extract queries, honest reveal queries, corrupt reveal queries and test queries made by $\mathcal{A}$, respectively. We say that a NIKE scheme is $\left(t, \epsilon, q_{H}, q_{C}, q_{E}, q_{H R}, q_{C R}, q_{T}\right)$-secure in the $m$-CKS-heavy model if there is no adversary with advantage at least $\epsilon$ that runs in time $t$ and makes at most $q_{H}$ register honest user ID queries, etc. Informally, we say that a NIKE scheme is $m$-CKS-heavy secure if there is no efficient adversary having nonnegligible advantage in $k$, where efficient means that the running time and numbers of queries made by the adversary are bounded by polynomials in $k$.

Comparing the models: Table 1 outlines the properties of our other security models in the DKR setting, in terms of restrictions on the queries that can be made by the adversary. It is apparent that the $m$-CKS-heavy model is the strongest model. It differs from the $C K S$-heavy model only in allowing multiple 
test queries. The $m$-CKS-heavy model represents a strengthening of the original $C K S$ model by allowing extract and honest reveal queries, whereas the $C K S$ model only allows the adversary to gain information about honestly generated shared keys via test queries. The $C K S$-light model is simplest of all, involving only two honestly registered identities, removing the extract and honest reveal queries, and allowing only a single test query. We prove that it is polynomially equivalent to the $m$-CKS-heavy model. In fact, we prove the following theorem:

Theorem 1. The m-CKS-heavy, CKS-heavy, CKS and CKS-light security models are all polynomially equivalent.

Proof. See the full version [25].

Thus, while the $m$-CKS-heavy model is our preferred model, it suffices to analyse schemes in the CKS-light model if one is not overly concerned about concrete security. However, we note that various factors are involved in the reductions. In particular a factor of $q_{T} q_{H}^{2}$ is lost in going from the $m$-CKS-heavy to the $C K S$ light model. This reflects the proof techniques used in establishing the bounds, specifically the use of hybrid arguments. It is an interesting open problem to either prove tighter relations between the models, or to prove that such results are not possible.

\section{Intractability Assumptions}

\subsection{The Group of Signed Quadratic Residues, the BBS generator, and the Strong Diffie-Hellman Assumption}

The factoring assumption: Let $n(k)$ be a function and $\delta$ a constant with $0 \leq \delta<$ $1 / 2$. Let RSAgen be an algorithm with input $1^{k}$ that generates elements $(N, P, Q)$ such that $N=P Q$ is an $n$-bit Blum integer and all prime factors of $\phi(N) / 4$ are pairwise distinct and have at least $\delta n$ bits. These conditions ensure that $\left(\mathbb{J}_{N}, \cdot\right)$ is cyclic and that the square $g$ of a random element in $\mathbb{Z}_{N}^{*}$, generates $\mathbb{Q R}_{N}$ with high probability. That is, $\langle g\rangle=\mathbb{Q R}_{N}$. For such $N$, we recall the definition of the group of signed quadratic residues $\mathbb{Q R}_{N}^{+}$from [20] (see also [26]27]) which is defined as the set $\left\{|x|: x \in \mathbb{Q R}_{N}\right\}$, where $|x|$ is the absolute value when representing elements of $\mathbb{Z}_{N}$ as the set $\{-(N-1) / 2, \ldots,(N-1) / 2\} \cdot\left(\mathbb{Q R}_{N}^{+}, \cdot\right)$ is a cyclic group of order $\phi(N) / 4$ whose elements are efficiently recognisable given only $N$ as input.

For any algorithm $\mathcal{A}$, we write

$$
\operatorname{Adv}_{\mathcal{A}, \mathrm{RSAgen}}^{\mathrm{fac}}(k)=\operatorname{Pr}\left[\{P, Q\} \stackrel{\$}{\leftarrow} \mathcal{A}(N):(N, P, Q) \stackrel{\$}{\leftarrow} \operatorname{RSAgen}\left(1^{\mathrm{k}}\right)\right]
$$

The factoring assumption for RSAgen is that $\operatorname{Adv}_{\mathcal{A}, \mathrm{RSAgen}}^{\mathrm{fac}}(k)$ is negligible for all PPT algorithms $\mathcal{A}$. 
The BBS generator: Let $\mathrm{BBS}_{N}: \mathbb{Q R}_{N}^{+} \rightarrow\{0,1\}^{k}$ be the Blum-Blum-Shub pseudorandom number generator. (That is, $\operatorname{BBS}_{N}(X)=\left(\operatorname{lsb}_{N}(X), \operatorname{lsb}_{N}\left(X^{2}\right), \ldots\right.$, $\left.\operatorname{lsb}_{N}\left(X^{2^{k-1}}\right)\right)$, where $\operatorname{lsb}_{N}(X)$ denotes the least significant bit of $X \in \mathbb{Q R}_{N}^{+}$.) Recall that the factoring assumption implies the computational indistinguishability of the distributions

$$
\left(N, X^{2^{k}}, \operatorname{BBS}_{N}(X)\right) \text { and }\left(N, X^{2^{k}}, R\right),
$$

where $N \stackrel{\$}{\leftarrow} \operatorname{RSAgen}\left(1^{\mathrm{k}}\right)$, and $X \stackrel{\$}{\leftarrow} \mathbb{Q R}_{N}^{+}$and $R \stackrel{\$}{\leftarrow}\{0,1\}^{k}$ are chosen uniformly. (See also [28, Theorem 2] for a summary why this holds.) Concretely, under the factoring assumption, the advantage

$$
\operatorname{Adv}_{\mathcal{B}, \text { RSAgen }}^{\mathrm{BBS}}(k):=\left|\operatorname{Pr}\left[\mathcal{B}\left(N, X^{2^{k}}, \operatorname{BBS}_{N}(X)\right)=1\right]-\operatorname{Pr}\left[\mathcal{B}\left(N, X^{2^{k}}, R\right)=1\right]\right|
$$

is negligible for any PPT adversary $\mathcal{B}$.

The Strong DH assumption: In [20] it is shown that if the factoring assumption holds, then the Strong DH assumption holds relative to RSAgen. This assumption is that there is no PPT algorithm having non-negligible advantage in solving the $\mathrm{CDH}$ problem on input $(N, g, X, Y)$ when given an oracle for $\mathrm{DDH}_{g, X}(\cdot, \cdot)$. Here $g$ is a randomly selected generator of $\mathbb{Q R}_{N}^{+}, X$ and $Y$ are selected uniformly from $\mathbb{Q} \mathbb{R}_{N}^{+}$, the solution to the CDH problem is defined as $g^{\left(\operatorname{dlog}_{g} X\right)\left(\operatorname{dlog}_{g} Y\right)}$, and the DDH oracle $\operatorname{DDH}_{g, X}(\hat{Y}, \hat{Z})$ returns 1 if $\hat{Y}^{\mathrm{dlog}_{g} X}=\hat{Z}$ and 0 otherwise.

We will require a variant of the Strong DH assumption, which we name the Double Strong DH (DSDH) assumption. This can be stated as follows. Let $(N, P, Q) \leftarrow \operatorname{RSAgen}\left(1^{\mathrm{k}}\right)$ and let $g$ be a randomly selected generator of $\mathbb{Q R}_{N}^{+}$, and $X, Y$ be selected uniformly from $\mathbb{Q R}_{N}^{+}$. Then the Double Strong DH problem is to solve the $\mathrm{CDH}$ problem on input $(N, g, X, Y)$, that is to compute $g^{\left(\operatorname{dlog}_{g} X\right)\left(\operatorname{dlog}_{g} Y\right)}$, when given oracles for $\operatorname{DDH}_{g, X}(\cdot, \cdot)$ and $\operatorname{DDH}_{g, Y}(\cdot, \cdot)$. The DSDH assumption relative to RSAgen is that there is no PPT algorithm having non-negligible advantage in solving this problem.

Theorem 2. If the factoring assumption holds relative to RSAgen, then the $D S D H$ assumption also holds relative to RSAgen. In particular, for every algorithm $\mathcal{A}$ solving the Double Strong DH problem, there exists a factoring algorithm $\mathcal{B}$ (with roughly the same running time as $\mathcal{A}$ ) such that

$$
\operatorname{Adv}_{\mathcal{A}, \text { RSAgen }}^{\text {dsdh }}(k) \leq \operatorname{Adv}_{\mathcal{B}, \text { RSAgen }}^{\mathrm{fac}}(k)+O\left(2^{-\delta n(k)}\right) .
$$

Proof. The original proof of [20, Theorem 2] shows how to handle a single DDH oracle $\operatorname{DDH}_{g, X}(\cdot, \cdot)$. By symmetry of the set-up used in the proof, the same procedure can also be used to (simultaneously) handle the oracle $\mathrm{DDH}_{g, Y}(\cdot, \cdot)$.

\subsection{Parameter Generation Algorithms for Asymmetric Pairings}

Our pairing based scheme will be parameterized by a type 2 pairing parameter generator, denoted by $\mathcal{G} 2$. This is a polynomial time algorithm that on input 
a security parameter $1^{k}$, returns the description of three multiplicative cyclic groups $\mathbb{G}_{1}, \mathbb{G}_{2}$ and $\mathbb{G}_{T}$ of the same prime order $p$, generators $g_{1}, g_{2}$ for $\mathbb{G}_{1}, \mathbb{G}_{2}$ respectively, and a bilinear non-degenerate and efficiently computable pairing $e$ : $\mathbb{G}_{1} \times \mathbb{G}_{2} \rightarrow \mathbb{G}_{T}$. We assume that $\mathcal{G} 2$ also outputs the description of an efficiently computable isomorphism $\psi: \mathbb{G}_{2} \rightarrow \mathbb{G}_{1}$ and that $g_{1}=\psi\left(g_{2}\right)$. Throughout, we write $\mathcal{P} \mathcal{G} 2=\left(\mathbb{G}_{1}, \mathbb{G}_{2}, \mathbb{G}_{T}, g_{1}, g_{2}, p, e, \psi\right)$ for a set of groups and other parameters with the properties just described.

\subsection{The Decisional Bilinear Diffie-Hellman Assumption for Type 2 Pairings (DBDH-2)}

Let $\mathcal{P} \mathcal{G} 2=\left(\mathbb{G}_{1}, \mathbb{G}_{2}, \mathbb{G}_{T}, g_{1}, g_{2}, p, e, \psi\right)$ as above. We consider the following version of the Decisional Bilinear Diffie-Hellman problem for type 2 pairings, as introduced by Galindo in [29]: Given $\left(g_{2}, g_{2}^{a}, g_{2}^{b}, g_{1}^{c}, T\right) \in \mathbb{G}_{2}^{3} \times \mathbb{G}_{1} \times \mathbb{G}_{T}$ as input, the problem is to decide whether or not $T=e\left(g_{1}, g_{2}\right)^{a b c}$, where $g_{1}=\psi\left(g_{2}\right)$. More formally, we associate the following experiment to a type 2 pairing parameter generator $\mathcal{G} 2$ and an adversary $\mathcal{B}$.

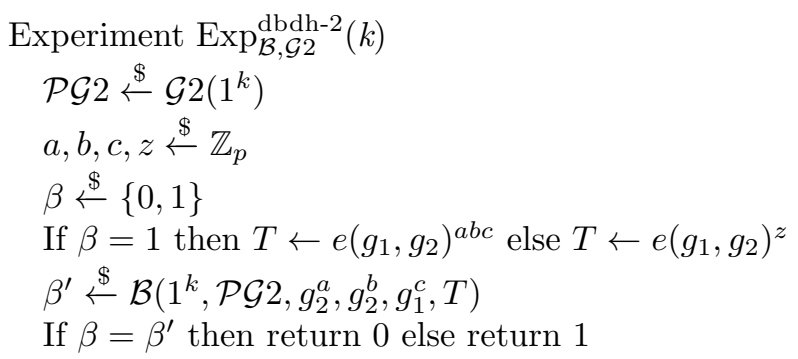

The advantage of $\mathcal{B}$ in the above experiment is defined as

$$
\operatorname{Adv}_{\mathcal{B}, \mathcal{G} 2}^{\mathrm{dbdh}-2}(k)=\left|\operatorname{Pr}\left[\operatorname{Exp}_{\mathcal{B}, \mathcal{G} 2}^{\mathrm{dbdh}-2}(k)=1\right]-\frac{1}{2}\right| .
$$

We say that the DBDH-2 assumption relative to $\mathcal{G} 2$ holds if $\mathrm{Adv}_{\mathcal{B}, \mathcal{G} 2}^{\mathrm{dbdh}-2}$ is negligible in $k$ for all PPT algorithms $\mathcal{B}$.

\section{Constructions for Non-Interactive Key Exchange}

\subsection{A Construction in the Random Oracle Model from Factoring}

We specify how to build a NIKE scheme, NIKE $_{\mathrm{fac}}$, that is secure in the CKS-light security model under the factoring assumption relative RSAgen in the ROM. Our scheme makes use of a hash function $H:\{0,1\}^{*} \rightarrow\{0,1\}^{k}$ which is modelled as a random oracle in the security proof. The component algorithms of the scheme $\mathrm{NIKE}_{\mathrm{fac}}$ are defined as follows: 


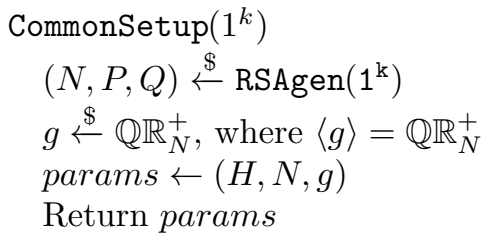

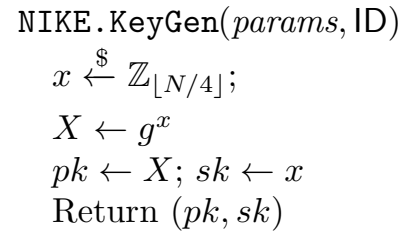

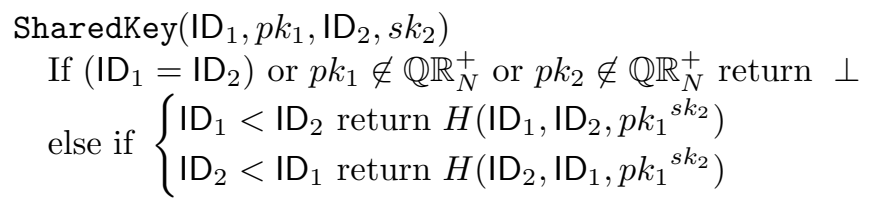

Here we are assuming that the identities ID come from a space with a natural ordering $<$.

Theorem 3. The scheme $\mathrm{NIKE}_{\mathrm{fac}}$ is secure in the ROM under the factoring assumption relative to RSAgen. In particular, suppose $\mathcal{A}$ is an adversary against NIKE $_{\mathrm{fac}}$ in the CKS-light security model. Then there exists a factoring adversary $\mathcal{C}$ with:

$$
\operatorname{Adv}_{\mathcal{A}, \mathrm{NIKE}_{\mathrm{fac}}}^{\text {CKS-light }}(k) \leq \operatorname{Adv}_{\mathcal{C}, \text { RSAgen }}^{\mathrm{fac}}(k)+O\left(2^{-\delta n(k)}\right) .
$$

Proof. See the full version [25].

\subsection{Towards a Factoring-Based Scheme in the Standard Model}

The security proof of NIKE $_{\mathrm{fac}}$ above crucially uses the statistical properties of the random oracle $H$. If we accept an interactive key registration, we can however give a factoring-based NIKE scheme in the standard model. The basis of this scheme is the factoring-based IND-CCA secure encryption scheme of Hofheinz and Kiltz 28. However, in adapting their scheme to the NIKE setting, we will have to find a way to simultaneously cope with two challenge ciphertexts (which correspond to the public keys of the challenge identities). To cope with this modified setting, we will set up a simulation that is able to decrypt all but two ciphertexts (resp. NIKE public keys).

In our description, let RSAgen as before, let ChamH : $\{0,1\}^{*} \times \mathcal{R}_{\mathrm{Cham}} \rightarrow$ $\mathbb{Z}_{2^{k}}$ be a chameleon hash function 22 . Now consider the following scheme NIKE fac-int: $_{\text {f }}$ :

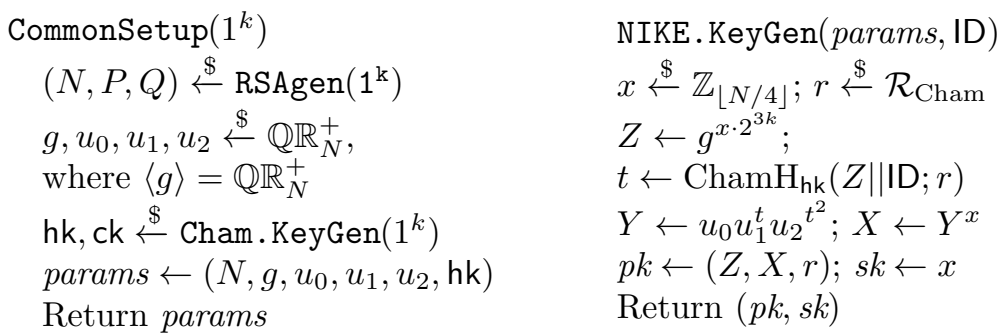




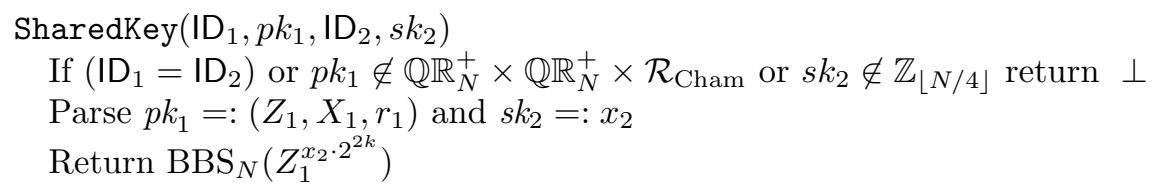

Note that correctness of the scheme follows from $Z_{1}^{x_{2} \cdot 2^{2 k}}=g^{x_{1} \cdot x_{2} \cdot 2^{5 k}}=Z_{2}^{x_{1} \cdot 2^{2 k}}$. To prove security, we need to rely on the consistency of public keys. Concretely, the security reduction we will give can only authentically answer corrupt reveal queries for corrupt user keys $p k=(Z, X, r)$ that satisfy $Z=g^{x \cdot 2^{3 k}}, X=$ $\left(u_{0} u_{1}^{t} u_{2}^{t^{2}}\right)^{x}$ for $t=\mathrm{ChamH}_{\mathrm{hk}}(Z|| \mathrm{ID} ; r)$ and some $x$. Unlike in our upcoming pairing-based scheme, this kind of consistency is not (obviously) efficiently verifiable. Hence, the key registration process must ensure that only consistent user keys are registered, e.g., by having the user prove consistency in zero-knowledge (interactively, using $x$ as witness).

On top of assuming consistent keys, we will also have to make an assumption about the distribution of (or rather, the ability to generate) primes. Namely, we will need to assume a PPT algorithm PrimeGen that, on input a $2 k$-bit prime $\rho$, outputs a prime $\alpha$ such that $\alpha \bmod \rho$ has statistical distance $O\left(2^{-k}\right)$ from the uniform distribution over $\mathbb{Z}_{\rho}$. Such an algorithm PrimeGen exists. This is an easy consequence of Dirichlet's theorem on the distribution of primes in arithmetic progressions: our generator simply samples integers of the form $\alpha_{0}+i \cdot \rho$ for uniformly chosen $\alpha_{0} \in \mathbb{Z}_{\rho}$ and $i=1,2, \ldots$, and checks them for primality. This algorithm can be rigorously proven to be efficient under the Generalized Riemann Hypothesis.

Theorem 4. Under the factoring assumption relative to RSAgen, given an algorithm PrimeGen as above, and assuming that the chameleon hash function ChamH is collision-resistant, the scheme $\mathrm{NIKE}_{\mathrm{fac}-\mathrm{int}}$ is secure against all adversaries that only register consistent (in the sense above) user keys. In particular, suppose $\mathcal{A}$ is such an adversary against $\mathrm{NIKE}_{\mathrm{fac}}$ in the $\mathrm{CKS}$-light security model. Then there exists a BBS distinguisher $\mathcal{B}$ and a collision-finder $\mathcal{A}_{\mathcal{C H}}$ with:

$$
\operatorname{Adv}_{\mathcal{A}, \text { NIKE }_{\text {fac-int }}}^{\text {CKS-light }}(k) \leq \operatorname{Adv}_{\mathcal{B}, \text { RSAgen }}^{\mathrm{BBS}}(k)+\operatorname{Adv}_{\mathcal{A}_{\mathcal{C H}}, \mathrm{ChamH}}^{\text {coll }}(k)+O\left(2^{-k}\right) .
$$

Proof. See the full version [25].

\subsection{A Construction in the Standard Model from Pairings}

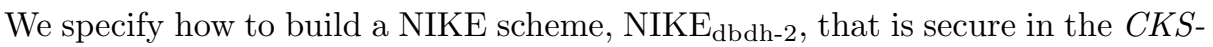
light security model under the DBDH-2 assumption in the standard model. Our construction makes use of a tuple $\mathcal{P} \mathcal{G} 2=\left(\mathbb{G}_{1}, \mathbb{G}_{2}, \mathbb{G}_{T}, g_{1}, g_{2}, p, e, \psi\right)$, output by a parameter generator $\mathcal{G} 2$, and a chameleon hash function $\mathrm{ChamH}:\{0,1\}^{*} \times$ $\mathcal{R}_{\text {Cham }} \rightarrow \mathbb{Z}_{p}$. This can be instantiated efficiently using the discrete-log based construction from [22]. The component algorithms of the scheme $\mathrm{NIKE}_{\mathrm{dbdh}-2}$ are defined as follows: 

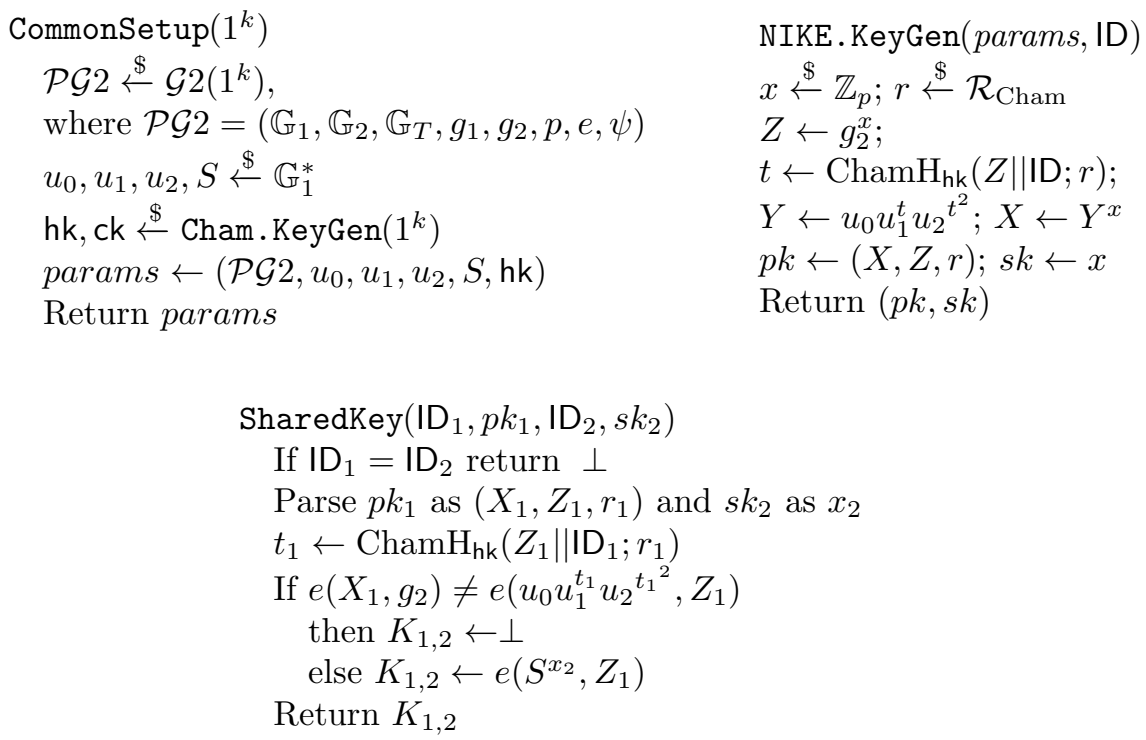

The check in the SharedKey algorithm for valid public keys can be implemented by evaluating the bilinear map twice. It is clear that SharedKey defined in this way satisfies the requirement that entities $\mathrm{ID}_{1}$ and $\mathrm{ID}_{2}$ are able to compute a common key. To see this, note that $e\left(S^{x_{2}}, Z_{1}\right)=e\left(S, g_{2}\right)^{x_{1}, x_{2}}$. The identity space for this construction, $\mathcal{I D S}$, is $\{0,1\}^{*}$, while the space of shared keys is $\mathcal{S H K}=\mathbb{G}_{T}$. Public keys and parameters are compact. For example, at the 128bit security level, using BN curves [30] and point compression, public keys consist of 768 bits plus an element from $\mathcal{R}_{\text {Cham }}$.

As stated before, we can prove the above NIKE scheme to be secure under the DBDH-2 assumption in the sense of the CKS-light security model. Interestingly, our scheme can be generalised to use any weak (2, poly)-PHF [21] in combination with a chameleon hash function. That is, $Y$ (in the NIKE.KeyGen algorithm) would be the output of the weak (2, poly)-PHF on input $t$, where $t$ is the output of the chameleon hash function. We have given a specific construction here because suitable weak PHFs are currently rare. A further generalisation of our scheme could use any randomised (2, poly)-PHF and avoid the chameleon hash, but no constructions for these are currently known.

Theorem 5. Assume ChamH is a family of chameleon hash functions. Then $\mathrm{NIKE}_{\mathrm{dbdh}-2}$ is secure under the DBDH-2 assumption relative to generator $\mathcal{G} 2$. In particular, suppose $\mathcal{A}$ is an adversary against $\mathrm{NIKE}_{\mathrm{dbdh}-2}$ in the CKS-light security model. Then there exists a DBDH-2 adversary $\mathcal{B}$ with:

$$
\operatorname{Adv}_{\mathcal{B}, \mathcal{G} 2}^{\mathrm{dbdh}-2}(k) \geq \operatorname{Adv}_{\mathcal{A}, \mathrm{NIKE}_{\mathrm{dbdh}-2}}^{\mathrm{CKS}-\text { light }}(k)-\operatorname{Adv}_{\mathcal{A}_{\mathcal{C} \mathcal{H}}, \mathrm{ChamH}}^{\text {coll }}(k)
$$

Proof. See the full version [25]. 


\section{From Non-Interactive Key Exchange to Public Key Encryption}

We give a conversion that takes a NIKE scheme that is secure in the CKS-light security model plus a strongly one-time secure signature (OTS) scheme, and produces from it a KEM that is IND-CCA secure. From such a KEM, it is easy to construct an IND-CCA secure public key encryption scheme [31.

The formal definitions of KEM and OTS schemes and their security can be found in the full version 25 .

\subsection{The Conversion from NIKE to KEM}

We now present our conversion from a NIKE scheme to a KEM. For a NIKE scheme NIKE and an OTS scheme OTS, we construct a KEM KEM(NIKE, OTS) with the following algorithms:

- KEM.KeyGen $\left(1^{k}\right)$ : This algorithm runs the algorithm CommonSetup $\left(1^{k}\right)$ of NIKE to obtain a set of system parameters, params. Then it picks ID $\in \mathcal{I D} \mathcal{S}$ uniformly and runs NIKE. KeyGen (params, ID) to obtain a key pair $(p k, s k)$. It sets $p k_{\mathrm{KEM}}=($ params, ID,$p k)$ and $s k_{\mathrm{KEM}}=(\mathrm{ID}, s k)$.

- Enc $\left(p k_{\mathrm{KEM}}\right)$ : This algorithm parses $p k_{\mathrm{KEM}}$ as (params, ID, $p k$ ), runs OTSKeyGen to obtain a pair $(v k, s i g k)$. This is repeated until $v k \neq$ ID. Next, it runs NIKE.KeyGen (params, ID' $=v k)$ of NIKE to obtain a key pair $\left(p k^{\prime}, s k^{\prime}\right)$ and runs OTSSign $\left(s i g k, p k^{\prime}\right)$ to obtain $\sigma$, a signature on $p k^{\prime}$. It then runs SharedKey (ID, $p k$, ID $\left.^{\prime}=v k, s k^{\prime}\right)$ of scheme NIKE to obtain a key $K \in \mathcal{S H K}$. The output is $\left(K, C=\left(v k, p k^{\prime}, \sigma\right)\right)$.

$-\operatorname{Dec}\left(s k_{\mathrm{KEM}}, C\right)$ : This algorithm first parses $C$ as $\left(v k, p k^{\prime}, \sigma\right)$ and $s k_{\mathrm{KEM}}$ as $(I D, s k)$. Next, it runs OTSVfy $\left(v k, p k^{\prime}, \sigma\right)$ and returns $\perp$ if the output is reject or if $v k=I D$. Otherwise, it runs SharedKey $\left(\mathrm{ID}^{\prime}=v k, p k^{\prime}, \mathrm{ID}, s k\right)$ and outputs the result, which may be $\perp$.

Notice that the ciphertexts in this scheme consist of a verification key from the OTS scheme, a public key from the NIKE scheme, and a one-time signature, while the encapsulated keys are elements of $\mathcal{S H K}$. As our next result shows, the resulting KEM is automatically IND-CCA secure if the NIKE scheme is secure in the CKS-light security model.

Theorem 6. Suppose the NIKE scheme NIKE is secure in the CKS-light security model and OTS is a strongly secure one-time signature scheme. Then $\mathrm{KEM}$ (NIKE, OTS) is an IND-CCA secure KEM. More precisely, for any adversary $\mathcal{A}$ against KEM(NIKE,OTS), there exists an adversary $\mathcal{B}$ against NIKE in the CKS-light security model or an adversary $\mathcal{C}$ against OTS having the same advantage. Moreover, if $\mathcal{A}$ makes $q_{D}$ decapsulation queries, then $\mathcal{B}$ makes $q_{D}$ register corrupt user queries and $q_{D}$ corrupt reveal queries, while $\mathcal{B}$ 's running time is roughly the same as that of $\mathcal{A}$.

Proof. See the full version [25]. 
Applying the above construction to the pairing-based NIKE scheme from the previous section results in an IND-CCA secure KEM with public keys (ID, $p k$ ) that consist of an identity string, two group elements (one in $\mathbb{G}_{1}$ and one in $\mathbb{G}_{2}$ ), and a key for the Chameleon hash function. Ciphertexts are slightly longer, containing in addition a verification key and a signature from the one-time signature scheme.

\section{Conclusions and Open Problems}

We provided different security models for NIKE and explored the relationships between them. We then gave constructions for secure NIKE in the ROM and in the standard model. We also studied the relationship between NIKE and PKE, showing that a secure NIKE implies an IND-CCA secure PKE scheme.

There are several interesting open problems that arise from our work. One is to construct pairing-free NIKE schemes in the standard model. A challenge to doing so is that our pairing-based construction uses the pairing in a fundamental way in order to provide a publicly computable check on the validity of public keys. The RSA/factoring setting seems particularly challenging in this respect - we recall that our standard model, factoring-based scheme required that the adversary only register valid public keys, a condition that could be enforced in practice by having an interactive key registration protocol and insisting on proofs of validity during that protocol. Clearly, it is desirable from both a practical and a theoretical perspective to obtain schemes that are secure in the plain setting, where no such protocol is required.

Another open problem is to construct ID-based NIKE schemes that are provably secure in the standard model, moving beyond the ROM schemes analysed in 810. Starting with known IBE schemes may be profitable, but the fact that these generally have randomised private key generation algorithms seems to make it hard to work backwards from IBE to ID-based NIKE.

Finally, it would be interesting to consider three-party NIKE schemes based on Joux's protocol 32. Currently, there is no security model for such schemes, and no constructions which can handle adversarially-generated public keys.

\section{References}

1. Diffie, W., Hellman, M.E.: New directions in cryptography. IEEE Transactions on Information Theory 22(6), 644-654 (1976)

2. Çapar, Ç., Goeckel, D., Paterson, K.G., Quaglia, E.A., Towsley, D., Zafer, M.: Signal-flow-based analysis of wireless security protocols. Information and Computation (to appear)

3. Dodis, Y., Katz, J., Smith, A., Walfish, S.: Composability and On-Line Deniability of Authentication. In: Reingold, O. (ed.) TCC 2009. LNCS, vol. 5444, pp. 146-162. Springer, Heidelberg (2009)

${ }^{3}$ Arguably, one might also include the public parameters params when evaluating the public key size. 
4. Boyd, C., Mao, W., Paterson, K.G.: Key Agreement Using Statically Keyed Authenticators. In: Jakobsson, M., Yung, M., Zhou, J. (eds.) ACNS 2004. LNCS, vol. 3089, pp. 248-262. Springer, Heidelberg (2004)

5. Jakobsson, M., Sako, K., Impagliazzo, R.: Designated Verifier Proofs and Their Applications. In: Maurer, U.M. (ed.) EUROCRYPT 1996. LNCS, vol. 1070, pp. 143-154. Springer, Heidelberg (1996)

6. Cash, D., Kiltz, E., Shoup, V.: The Twin Diffie-Hellman Problem and Applications. In: Smart, N.P. (ed.) EUROCRYPT 2008. LNCS, vol. 4965, pp. 127-145. Springer, Heidelberg (2008)

7. Sakai, R., Ohgishi, K., Kasahara, M.: Cryptosystems based on pairing. In: The 2000 Symposium on Cryptography and Information Security, pp. 26-28 (2000)

8. Dupont, R., Enge, A.: Provably secure non-interactive key distribution based on pairings. Discrete Applied Mathematics 154(2), 270-276 (2006)

9. Gennaro, R., Halevi, S., Krawczyk, H., Rabin, T., Reidt, S., Wolthusen, S.D.: Strongly-Resilient and Non-interactive Hierarchical Key-Agreement in MANETs. In: Jajodia, S., Lopez, J. (eds.) ESORICS 2008. LNCS, vol. 5283, pp. 49-65. Springer, Heidelberg (2008)

10. Paterson, K.G., Srinivasan, S.: On the relations between non-interactive key distribution, identity-based encryption and trapdoor discrete log groups. Des. Codes Cryptography 52(2), 219-241 (2009)

11. Bellare, M., Rogaway, P.: Entity Authentication and Key Distribution. In: Stinson, D.R. (ed.) CRYPTO 1993. LNCS, vol. 773, pp. 232-249. Springer, Heidelberg (1994)

12. Shoup, V.: On formal models for secure key exchange (version 4) (1999)

13. Bellare, M., Pointcheval, D., Rogaway, P.: Authenticated Key Exchange Secure against Dictionary Attacks. In: Preneel, B. (ed.) EUROCRYPT 2000. LNCS, vol. 1807, pp. 139-155. Springer, Heidelberg (2000)

14. Canetti, R., Krawczyk, H.: Analysis of Key-Exchange Protocols and Their Use for Building Secure Channels. In: Pfitzmann, B. (ed.) EUROCRYPT 2001. LNCS, vol. 2045, pp. 453-474. Springer, Heidelberg (2001)

15. Krawczyk, H.: HMQV: A High-Performance Secure Diffie-Hellman Protocol. In: Shoup, V. (ed.) CRYPTO 2005. LNCS, vol. 3621, pp. 546-566. Springer, Heidelberg (2005)

16. Bellare, M., Neven, G.: Multi-signatures in the plain public-key model and a general forking lemma. In: Juels, A., Wright, R.N., di Vimercati, S.D.C. (eds.) ACM Conference on Computer and Communications Security, pp. 390-399. ACM (2006)

17. Ristenpart, T., Yilek, S.: The Power of Proofs-of-Possession: Securing Multiparty Signatures against Rogue-Key Attacks. In: Naor, M. (ed.) EUROCRYPT 2007. LNCS, vol. 4515, pp. 228-245. Springer, Heidelberg (2007)

18. Boldyreva, A.: Threshold Signatures, Multisignatures and Blind Signatures Based on the Gap-Diffie-Hellman-Group Signature Scheme. In: Desmedt, Y.G. (ed.) PKC 2003. LNCS, vol. 2567, pp. 31-46. Springer, Heidelberg (2002)

19. Lu, S., Ostrovsky, R., Sahai, A., Shacham, H., Waters, B.: Sequential Aggregate Signatures and Multisignatures Without Random Oracles. In: Vaudenay, S. (ed.) EUROCRYPT 2006. LNCS, vol. 4004, pp. 465-485. Springer, Heidelberg (2006)

20. Hofheinz, D., Kiltz, E.: The Group of Signed Quadratic Residues and Applications. In: Halevi, S. (ed.) CRYPTO 2009. LNCS, vol. 5677, pp. 637-653. Springer, Heidelberg (2009)

21. Hofheinz, D., Jager, T., Kiltz, E.: Short Signatures from Weaker Assumptions. In: Lee, D.H., Wang, X. (eds.) ASIACRYPT 2011. LNCS, vol. 7073, pp. 647-666. Springer, Heidelberg (2011) 
22. Krawczyk, H., Rabin, T.: Chameleon signatures. In: NDSS (2000)

23. Chatterjee, S., Sarkar, P.: Generalization of the Selective-ID Security Model for HIBE Protocols. In: Yung, M., Dodis, Y., Kiayias, A., Malkin, T. (eds.) PKC 2006. LNCS, vol. 3958, pp. 241-256. Springer, Heidelberg (2006)

24. ElGamal, T.: A public key cryptosystem and a signature scheme based on discrete logarithms. IEEE Transactions on Information Theory 31(4), 469-472 (1985)

25. Freire, E.S.V., Hofheinz, D., Kiltz, E., Paterson, K.G.: Non-interactive key exchange. Cryptology ePrint Archive, Report 2012/xxx (2012), http://eprint.iacr.org/

26. Goldwasser, S., Micali, S., Rackoff, C.: The knowledge complexity of interactive proof systems. SIAM Journal on Computing 18(1), 186-208 (1989)

27. Fischlin, R., Schnorr, C.P.: Stronger security proofs for RSA and Rabin bits. Journal of Cryptology 13(2), 221-244 (2000)

28. Hofheinz, D., Kiltz, E.: Practical Chosen Ciphertext Secure Encryption from Factoring. In: Joux, A. (ed.) EUROCRYPT 2009. LNCS, vol. 5479, pp. 313-332. Springer, Heidelberg (2009)

29. Galindo, D.: Boneh-Franklin Identity Based Encryption Revisited. In: Caires, L., Italiano, G.F., Monteiro, L., Palamidessi, C., Yung, M. (eds.) ICALP 2005. LNCS, vol. 3580, pp. 791-802. Springer, Heidelberg (2005)

30. Barreto, P.S.L.M., Naehrig, M.: Pairing-Friendly Elliptic Curves of Prime Order. In: Preneel, B., Tavares, S. (eds.) SAC 2005. LNCS, vol. 3897, pp. 319-331. Springer, Heidelberg (2006)

31. Cramer, R., Shoup, V.: Design and analysis of practical public-key encryption schemes secure against adaptive chosen ciphertext attack. SIAM Journal on Computing 33, 167-226 (2003)

32. Joux, A.: A One Round Protocol for Tripartite Diffie-Hellman. In: Bosma, W. (ed.) ANTS 2000. LNCS, vol. 1838, pp. 385-394. Springer, Heidelberg (2000) 